\title{
A comparison of current serum biomarkers as diagnostic indicators of mitochondrial diseases OPEN
}

Ryan L. Davis, PhD

Christina Liang, MBBS

Carolyn M. Sue, MBBS, $\mathrm{PhD}$

Correspondence to

Dr. Sue:

carolyn.sue@sydney.edu.au

\section{ABSTRACT}

Objective: To directly compare the diagnostic utility of growth differentiation factor-15 (GDF-15) with our previous fibroblast growth factor-21 (FGF-21) findings in the same adult mitochondrial disease cohort.

Methods: Serum GDF-15 levels were measured using a quantitative ELISA. Statistical analyses of GDF-15 data were compared with our published FGF-21 findings.

Results: Median serum GDF-15 concentrations were elevated in patients with mitochondrial disease and differed between all experimental groups, mirroring group results for FGF-21. There was a difference between patients diagnosed by muscle biopsy and genetic diagnosis, suggesting that serum GDF-15 measurement may be more broadly specific for mitochondrial disease than for muscle manifesting mitochondrial disease, in contrast to FGF-21. GDF-15 showed a markedly higher diagnostic odds ratio when compared with FGF-21 (75.3 vs 45.7), was a better predictor of disease based on diagnostic sensitivity $(77.8 \%$ vs $68.5 \%)$, and outperformed FGF-21 on receiver operating characteristic curve analysis (area under the curve $94.1 \%$ vs $91.1 \%$ ). Combining both biomarkers did not improve the area under the curve remarkably over GDF-15 alone. GDF-15 was the best predictor of mitochondrial disease $(p<0.002)$ following multivariate logistic regression analysis.

Conclusions: GDF-15 outperforms FGF-21 as an indicator of mitochondrial diseases. Our data suggest that GDF-15 is generally indicative of inherited mitochondrial disease regardless of clinical phenotype, whereas FGF-21 seems to be more indicative of mitochondrial disease when muscle manifestations are present.

Classification of evidence: This study provides Class III evidence that serum GDF-15 accurately distinguishes patients with mitochondrial diseases from those without them. Neurology ${ }^{\circledR}$ 2016;86:2010-2015

\section{GLOSSARY}

AUC = area under the curve; $\mathbf{C I}$ = confidence interval; FGF-21 = fibroblast growth factor-21; GDF-15 = growth differentiation factor-15; IQR = interquartile range; MELAS = mitochondrial myopathy, encephalopathy, lactic acidosis, and strokelike episodes; $\mathbf{M R C}=$ Medical Research Council; NMDAS $=$ Newcastle Mitochondrial Disease Scale for Adults; OR = odds ratio; $\mathbf{R O C}$ = receiver operating characteristic; TK2 = thymidine kinase 2 gene.

The routine diagnosis of mitochondrial diseases is in part complicated by a distinct lack of sensitive serum biomarkers. Historically, insensitive markers such as creatine kinase, lactate, pyruvate, and the lactate:pyruvate ratio have been used, with minimal diagnostic utility. ${ }^{1-3}$ Recent efforts to identify more sensitive indicators of mitochondrial diseases led to the association of increased cellular expression and raised serum levels of fibroblast growth factor-21 (FGF-21) with mitochondrial myopathy in a mouse model. ${ }^{4}$ Subsequent studies in patient cohorts showed serum FGF-21 concentration to be a useful indicator of muscle manifesting mitochondrial disorders, greatly improving on the inference of classical disease markers. ${ }^{2,3}$ More recently, a gene

From the Department of Neurogenetics (R.L.D., C.M.S.), Kolling Institute of Medical Research, University of Sydney and Royal North Shore Hospital, Sydney, Australia; and the Department of Neurology (C.L., C.M.S), Royal North Shore Hospital, Sydney, Australia.

Go to Neurology.org for full disclosures. Funding information and disclosures deemed relevant by the authors, if any, are provided at the end of the article. The Article Processing Charge was paid by the authors.

This is an open access article distributed under the terms of the Creative Commons Attribution-NonCommercial-NoDerivatives License 4.0 (CC BY-NC-ND), which permits downloading and sharing the work provided it is properly cited. The work cannot be changed in any way or used commercially. 
expression study in humans with thymidine kinase 2 (TK2) gene mutations identified growth differentiation factor-15 (GDF-15) as a potential disease indicator. ${ }^{5} \mathrm{GDF}-15$ has since been reported to correlate with disease severity, but not progression, in patients with the m.3243A $>\mathrm{G}$ mutation ${ }^{6,7}$ and was found to reflect improvements in mitochondrial function following pyruvate supplementation in vitro. ${ }^{8}$ In light of recent reports, ${ }^{6-8}$ we present an analysis of the diagnostic capacity of serum GDF-15 levels to indicate mitochondrial disease, and compare it to our previously published data on FGF-21. ${ }^{2}$

METHODS Primary research question and classification of evidence. Is GDF-15 a better diagnostic indicator of mitochondrial diseases compared to FGF-21? This case-control study provides Class III evidence that GDF-15 is a better diagnostic indicator of mitochondrial diseases when compared with FGF-21 (diagnostic sensitivity, $77.8 \%$ vs $68.5 \%$; diagnostic odds ratio [OR], 73.5 vs 45.7 ; area under the receiver operating characteristic curve, 0.941 vs 0.911 ).

Study cohort and inclusion and exclusion criteria. The cohort used in this study was described previously. ${ }^{2}$ Briefly, the adult cohort consisted of 66 controls, 20 nonmitochondrial neuromuscular disease controls, and 54 consecutive patients with mitochondrial disease. Mitochondrial disease patients were recruited between November 2011 and October 2012 from the adult mitochondrial disease clinic at Royal North Shore Hospital, Sydney, Australia, if they met the Walker criteria ${ }^{9}$ for mitochondrial disease and had a confirmed genetic diagnosis or changes on muscle biopsy indicative of mitochondrial myopathy. Participants ranged in age from 20 to 84 years and $57.9 \%$ of participants were female. Of the 54 patients with mitochondrial disease, 27 had a genetic diagnosis and 27 had muscle biopsy changes indicative of a mitochondrial myopathy ( 9 patients with a genetic diagnosis also had a muscle biopsy showing changes consistent with mitochondrial myopathy). We refer to the Standards for Reporting of Diagnostic Accuracy diagram published previously for this cohort for inclusion and exclusion details. ${ }^{2}$ When possible, the Newcastle Mitochondrial Disease Scale for Adults (NMDAS) $)^{10}$ clinical rating scale was performed on patients with mitochondrial disease. In addition, patients were routinely assessed for proximal muscle weakness according to Medical Research Council (MRC) criteria. ${ }^{11}$

Standard protocol approvals, registrations, and patient consents. Written informed consent was obtained from all participants at the time of sample collection and ethical approval was granted by the Northern Sydney Local Health District Human Research Ethics Committee.

Biochemical assays. Serum creatine kinase, lactate, and pyruvate levels (along with other biochemical parameters) measured previously ${ }^{2}$ were used here for analysis with serum GDF-15 levels.

Serum GDF-15 measurement. GDF-15 levels were measured in archived serum samples by quantitative sandwich ELISA (BioVendor, Laboratorni Medicina a.s., Brno, Czech Republic), according to the manufacturer's instructions. Absorbance readings at $570 \mathrm{~nm}$ were subtracted from absorbance readings at $450 \mathrm{~nm}$ before normalizing to the mean absorbance of blank wells. Triplicate measurements for each sample were averaged and the concentration determined from a 4-parameter logistic standard curve (www.myassays.com). ELISA measurements were performed in triplicate for each assay and repeated in at least duplicate assays. The scientist who performed the assays (R.L.D.) was blinded to samples and their groupings.

Statistical analysis. Statistical analyses were performed, as previously described, ${ }^{2}$ using SPSS version 20 (IBM Corporation, Armonk, NY) and clinical research calculators on the Vassar University statistics Web interface (http://vassarstats.net/). Differences between groups were considered statistically significant when 2 -tailed $p$ values were less than 0.05 . All $p$ values below 0.0001 are represented as $p<0.0001$. The reference cutoff concentration for GDF-15 was set at the 95 th percentile of control group serum concentrations, as for FGF-21. ${ }^{2}$

We used Mann-Whitney $U$ and Kruskal-Wallis nonparametric testing to determine statistical differences between groups. We calculated the diagnostic sensitivity and the corresponding $95 \%$ confidence intervals. An unadjusted diagnostic OR was determined for comparison with FGF-21. Receiver operating characteristic (ROC) curves were plotted using sensitivity vs $100-$ specificity on a continuous scale and the area under the curve (AUC) was used to determine the relative diagnostic capacity. Biomarker levels were correlated with other biochemical measurements and clinical assessment tools using nonparametric Spearman correlation testing. Linear regression analysis was used to determine if statistically correlated parameters could predict GDF-15 concentration. Multivariate linear regression analysis was performed to determine factors capable of predicting disease while controlling for confounders that may influence serum GDF-15 concentration.

RESULTS Median serum GDF-15 concentrations varied considerably among the 3 cohort groups: the control group had a median serum GDF-15 concentration of $1,183.5 \mathrm{pg} / \mathrm{mL}$ (interquartile range [IQR] 1,037.5-1,475.3), disease controls had a median concentration of $1,791.0 \mathrm{pg} / \mathrm{mL}$ (IQR 1,148.0-3,406.8), and patients with mitochondrial disease had a median concentration of $3,956.0 \mathrm{pg} / \mathrm{mL}$ (IQR 2,516.5-8,676.8). The interassay coefficient of variation was $9.6 \%$ for repeated samples ( 40 samples repeated in duplicate and 42 samples repeated in triplicate from across the 3 experimental groups) and $10.7 \%$ for assay quality controls. On average, serum GDF-15 concentrations were 2 and 4.6 times higher than control levels for the disease control and mitochondrial disease groups, respectively. When compared to mean FGF-21 levels, disease control and mitochondrial disease groups were 2.2 and 7.3 times higher than control levels, respectively. ${ }^{2}$ As with FGF-21 (threshold cutoff $350 \mathrm{pg} / \mathrm{mL}$ ), the 95th percentile of control subjects was set as the threshold cutoff for GDF-15 (threshold cutoff $2,330 \mathrm{pg} / \mathrm{mL}$ ) (figure 1). The same relationship in median biomarker concentration between groups was observed for both biomarkers and nonparametric Mann-Whitney $U$ testing showed serum biomarker concentrations to differ between the groups (figure 1). 
Figure 1 Box and whisker plots comparing fibroblast growth factor-21 (FGF-21) and growth differentiation factor-15 (GDF-15) concentrations between experimental groups

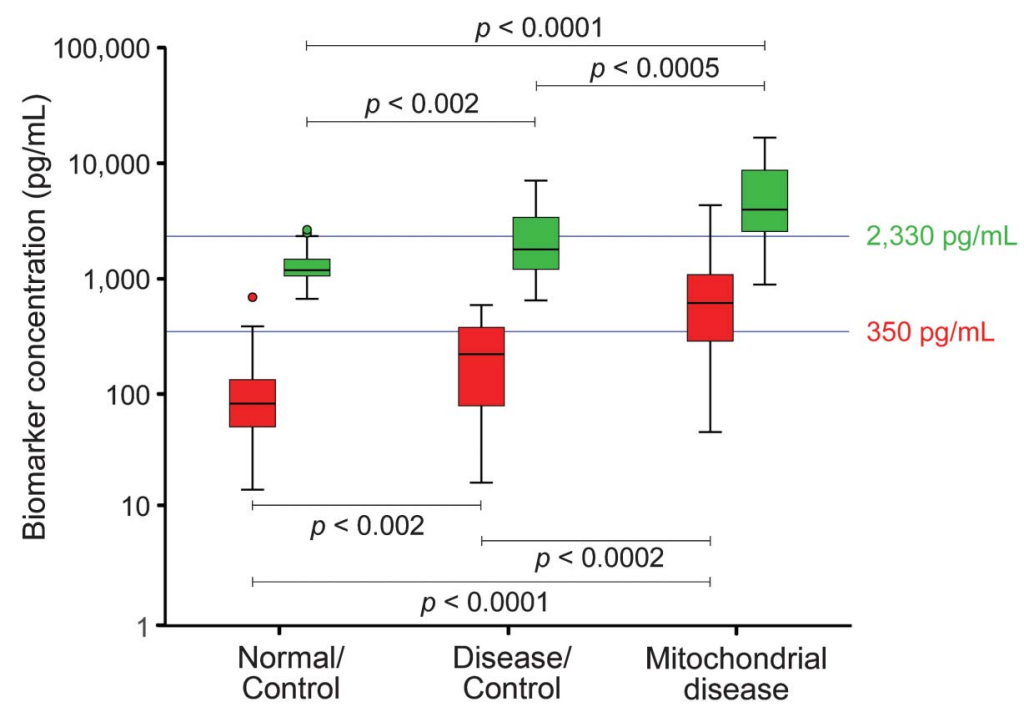

Serum FGF-21 (red) and GDF-15 (green) concentrations are displayed for the 3 experimental groups on a logarithmic scale. The same relationship between median group concentrations can be seen for both biomarkers, with higher levels in the mitochondrial disease group compared to disease controls and controls. Biomarker concentrations were compared between experimental groups using nonparametric Mann-Whitney $U$ testing. Threshold cutoffs (determined from the 95th percentile of control group biomarker concentrations) are indicated by blue horizontal lines at the level of $350 \mathrm{pg} / \mathrm{mL}$ for FGF-21 and 2,330 pg/mL for GDF-15.
In addition, Mann-Whitney $U$ (muscle biopsy vs genetic diagnosis, mtDNA vs nDNA mutation, muscle manifesting vs non-muscle manifesting, mitochondrial myopathy, encephalopathy, lactic acidosis, and strokelike episodes [MELAS] vs non-MELAS, male vs female, diabetic vs normal, cardiovascular involvement vs no cardiovascular involvement) and Kruskal-Wallis (Walker criteria [possible, probable, definite], diabetic status [normal, type I diabetes, type II diabetes, impaired fasting glucose, impaired glucose tolerance, insulin resistance], body mass index [anorexic, normal, obese], cardiovascular status [normal, cardiomyopathy, ischemic heart disease, rhythm disturbances, and periprocedural myocardial infarction, as well as cardiomyopathy, rhythm disturbances, and periprocedural myocardial infarction]) nonparametric analyses revealed only one statistical difference among all the groups and categories compared. The criterion of muscle biopsy diagnosis vs genetic diagnosis showed a marginal difference in serum GDF-15 concentration $(3,206 \mathrm{pg} / \mathrm{mL}$ [IQR $2,152-6,028]$ vs $6,415 \mathrm{pg} / \mathrm{mL}$ [IQR 2,704-12,345], respectively; $p=$ 0.046). Of note, in our previous FGF-21 study, there was a difference between muscle manifesting and non-muscle manifesting disease patients, indicative of FGF-21 elevation in myopathic disease states. ${ }^{2}$ This difference was not evident when analyzing GDF-15 ( $p=0.324)$, suggesting GDF-15 production may not be related to tissue-specific pathways, but rather pathways common to all tissues. This highlights a potentially broader indication of mitochondrial diseases by GDF-15 that is not evident for FGF-21.

The vast majority of literature discussing GDF-15 as a disease biomarker pertains to cardiovascular abnormalities, in particular ischemic heart disease. Although cardiac involvement is a relatively common comorbidity of mitochondrial disease, the majority of patients in this study did not have cardiac pathology. When analyzing GDF-15 against all cardiac involvement and also against subcategories of cardiac involvement, there was no apparent statistical difference in serum GDF-15 concentration between those with $(\mathrm{n}=11)$ or without $(\mathrm{n}=43)$ cardiac involvement $(p=0.788)$, or when subcategorized (KruskalWallis, $p=0.451)$.

Serum biomarker concentration exceeding the threshold cutoff was considered indicative of disease for statistical analyses. As the threshold cutoffs were set at the 95th percentile of control concentrations, the specificity was artificially determined to be 95.5\% (95\% confidence interval [CI] 86.4\%98.8\%) for FGF-21 and GDF-15 in both disease control and mitochondrial disease groups. The sensitivity for detecting mitochondrial disease if serum GDF-15 concentrations were raised was $77.8 \%$ (95\% CI $64.1 \%-87.5 \%$ ), as compared to FGF-21 at $68.5 \%$ (95\% CI $54.3 \%-80.0 \%)$. For the disease control group, the sensitivity for predicting mitochondrial disease when there was none was $30.0 \%$ (95\% CI 12.8\%-54.3\%) using GDF-15, compared to $35.0 \%$ (95\% CI $16.3 \%-59.1 \%$ ) using FGF-21. When subcategorizing the mitochondrial disease group by diagnosis, the sensitivity for patients diagnosed by muscle biopsy was 59.3\% (95\% CI 39.0\%$77.0 \%)$ using serum FGF-21 concentration and 70.4\% (95\% CI 49.7\%-85.5\%) using serum GDF15 concentration. For patients diagnosed with a genetic mutation, the sensitivity was $77.8 \%$ (95\% CI $57.3 \%-90.6 \%$ ) using serum FGF-21 concentration and $85.2 \%$ (95\% CI $65.4 \%-95.1 \%)$ using serum GDF-15 concentration. Nine of the patients with genetic diagnoses also had a muscle biopsy showing changes consistent with mitochondrial disease. The sensitivity when considering these patients was 66.7\% (95\% CI 30.92-90.96) using serum FGF-21 concentrations and $88.9 \%$ (95\% CI 50.67-99.42) using serum GDF-15 concentration.

The likelihood of having disease if FGF-21 levels were raised above the threshold cutoff of $350 \mathrm{pg} / \mathrm{mL}$, known as the diagnostic OR, was calculated in our previous study to be 45.7 (95\% CI 12.5-166.5, $p<$ $0.0001) .^{2}$ For GDF-15 in this study, the diagnostic OR was calculated to be 73.5 (95\% CI 19.6-276.3, 
$p<0.0001)$. Further to this, ROC curve analysis of FGF-21 and GDF-15 showed GDF-15 to be slightly better (AUC 0.941 [95\% CI 0.893-0.989]) than FGF-21 (AUC 0.911 [95\% CI 0.855-0.968]) at predicting disease across combinations of sensitivity and specificity, although the AUCs were not statistically different. Combining both biomarkers did not increase the AUC $(0.944$ [95\% CI 0.8950.994]) remarkably over that for GDF-15 alone, indicating that there was little synergistic diagnostic benefit when considering the biomarkers together.

Statistical dependence between GDF-15 and all other measured parameters from the initial study ${ }^{2}$ was assessed for the disease group using Spearman rank correlation coefficient test. GDF-15 correlated with lactate (Spearman correlation coefficient $\left[r_{s}\right]=$ 0.583, $p<0.0001)$, lactate:pyruvate $\left(r_{s}=0.544\right.$, $p<0.0001)$, FGF-21 $\left(r_{s}=0.554, p<0.0001\right)$, body mass index $\left(r_{s}=-0.322, p<0.02\right)$, MRC proximal muscle weakness ${ }^{11}\left(r_{s}=-0.302, p<0.05\right)$, and the Walker criteria9 $\left(r_{s}=0.322, p<0.02\right)$. Interestingly, unlike in other GDF-15 studies, ${ }^{6,712}$ those patients in the mitochondrial disease group for whom an $\mathrm{NMDAS}^{10}$ had been completed at the time of recruitment $(\mathrm{n}=30)$ showed no correlation between the total NMDAS score or scores for subsections (I-III) and serum FGF-2 $1^{2}$ or GDF-15 concentration.

Following multivariate linear regression analysis for each of the groups to determine any variables that could predict serum GDF-15 levels, only lactate: pyruvate $(p<0.002)$ and FGF-21 $(p<0.01)$ remained associated in the mitochondrial disease group. Despite a moderate correlation between GDF-15 and FGF-21 $\left(r_{s}=0.554\right)$, an association that persisted after linear regression, there was no benefit to disease prediction when the 2 biomarkers were combined for ROC curve analysis (figure 2), as mentioned above. Finally, when considering GDF-15 levels from control and mitochondrial disease groups in a multivariate logistic regression model that included possible confounders (age, lactate:pyruvate, insulin, glucose, cholesterol, triglycerides, and FGF-21), GDF-15 was the best predictor of mitochondrial disease $(p<0.002)$.

DISCUSSION In our cohort of adult patients with mitochondrial disease, GDF-15 presents as a sensitive indicator of mitochondrial diseases and outperforms FGF-21 and classical markers. Median serum concentrations of GDF-15 showed the same relationship between experimental groups as was observed for FGF-21 (figure 1), but GDF-15 was a better predictor of disease based on diagnostic sensitivity, diagnostic OR, ROC curves (figure 2), and multivariate linear regression. Despite a moderate correlation between serum GDF-15 and FGF-21 concentration, a relationship that persisted after linear regression analysis, there was no synergistic benefit for considering GDF-15 and FGF-21 together (figure 2). Furthermore, GDF-15 was more broadly indicative of mitochondrial diseases, rather than preferentially indicating muscle manifesting mitochondrial disease like FGF-21.

The design of the current study differs from previous studies in several ways. First, we surveyed an adult-only cohort of patients with mitochondrial disease, whereas other FGF-21 and GDF-15 diagnostic studies have looked at combined adult and pediatric cohorts. ${ }^{3,78}$ As pediatric patients are known to exhibit higher circulating biomarker levels, ${ }^{3,7}$ analyses combining these populations may artificially skew serum biomarker concentrations and therefore the analysis of results. Second, our cohort included a combination of patients with genetic and muscle histology diagnoses, representing current clinical diagnostic methods. As such, our study represents a more stringent estimation of biomarker diagnostic validity, compared to other studies that have considered only genetically diagnosed cohorts. ${ }^{3,6,7,12}$ Given our results, we propose that biomarker triaging of patients suspected of a mitochondrial disorder may prove to be a more efficient diagnostic paradigm when performed in combination with exhaustive genetic sequencing analysis using next-generation sequencing. ${ }^{13}$ Third, our cohort represents a wider range of mitochondrial diseases in comparison to other studies that have used limited genetic mitochondrial disease groups, such as m.3243A $>$ G, Kearns-Sayre syndrome, mitochondrial DNA deletions, or TK2 mutations. ${ }^{5-8}$ Finally, we used assay kits from the same manufacturer (FGF21 and GDF-15; BioVendor, Brno, Czech Republic), as compared to other studies that used assays from different manufacturers (FGF-21; BioVendor and GDF-15; R\&D Systems, Minneapolis, MN) ${ }^{6-8,12}$ to reduce potential variation in methodology and experimental output.

The median GDF-15 concentration for controls in this study $(1,183.5 \mathrm{pg} / \mathrm{mL}$ [IQR $1,037.5-$ $1,475.3])$ was in good agreement with median GDF-15 concentrations for controls $(1,020 \mathrm{pg} / \mathrm{mL}$ [IQR $803-1,362 \mathrm{pg} / \mathrm{mL}$ ] in men and $1,017 \mathrm{pg} / \mathrm{mL}$ [IQR $809-1,297 \mathrm{pg} / \mathrm{mL}$ ] in women) from the Framingham Offspring Study, ${ }^{14}$ despite differing methods of quantification. However, when age- and sex-matched 97.5th percentile values from the Framingham study were used as the threshold cutoff for a genetically diagnosed m.3243A $>G$ European patient cohort study, a diagnostic sensitivity of only $53 \%$ was calculated. ${ }^{6,14}$ By comparing the 97.5 th percentile values from the Framingham study (range $1,085-5,006 \mathrm{pg} / \mathrm{mL}$, age- and sex-dependent, ${ }^{14}$ using precommercial automated electrochemiluminescence 
Figure 2 Comparison of continuous-scale receiver operating characteristic curves for growth differentiation factor-15 (GDF-15) and fibroblast growth factor-21 (FGF-21), both individually and combined

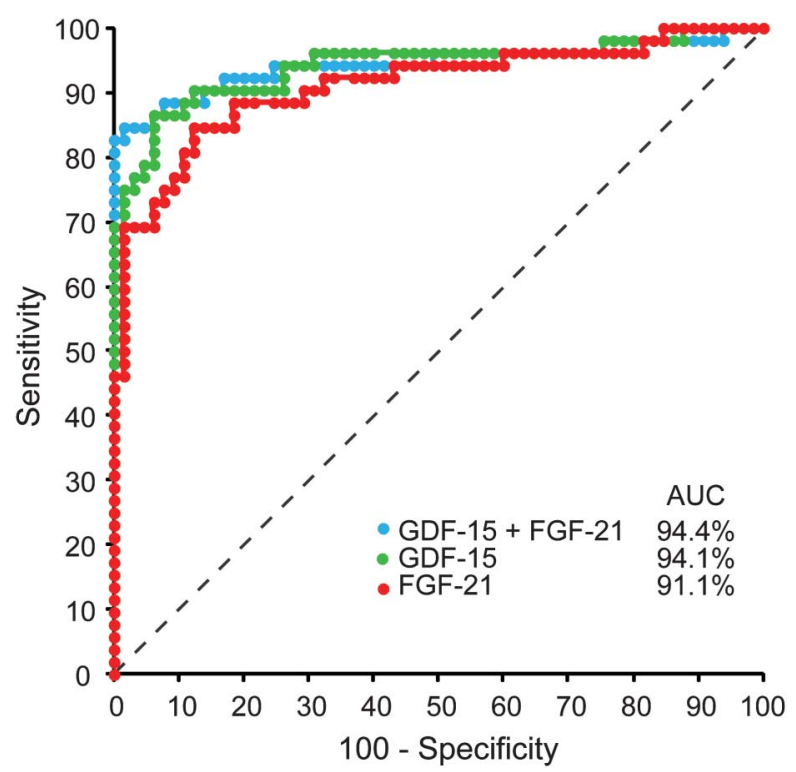

GDF-15 (green) outperformed FGF-21 (red) across varying sensitivities at high specificities (area under the curve $[\mathrm{AUC}]=0.941$ vs 0.911 , respectively; no difference). Combining GDF15 and FGF-21 (blue) had little benefit over GDF-15 alone (AUC $=0.944$ vs 0.941, respectively; no difference). Dotted line $=$ line of no discrimination ( $A U C=0.50$ ).

immunoassay) with the threshold cutoff of a recent Japanese mitochondrial disease patient GDF-15 diagnostic study $(710 \mathrm{pg} / \mathrm{mL})^{7}$ using R\&D systems Quantikine ELISA), obtained using the same ELISA assay as the European study, ${ }^{6}$ it becomes apparent that the use of different assays can give markedly different results and the use of data from one assay type in association with a different assay can potentially be misleading. Despite this, the correlation between serum GDF-15 and FGF-21 concentrations was in agreement between this study and the European study $\left(r_{s}=0.554\right.$ vs $r_{s}=$ 0.54 , respectively) and close to that reported in the Japanese study $\left(r_{s}=0.64\right){ }^{6,7}$

Our study demonstrates that elevated GDF-15 levels were indicative of mitochondrial disease regardless of whether there was muscle involvement. This is in contrast to the Japanese study, which claimed that GDF-15 may be a better indicator of muscle manifesting disease than FGF-21, even though a measure of muscle involvement was not used in that study and therefore comparisons relating muscle involvement with biomarker concentration were not possible. Furthermore, that study used different threshold values for GDF-15: they agreed with our previously determined FGF-21 threshold cutoff value of $350 \mathrm{pg} / \mathrm{mL}$, when using the same ELISA from BioVendor, ${ }^{2,7}$ but the threshold cutoff values for GDF-15 were set at a lower level $\left(\mathrm{R} \& \mathrm{D}\right.$ Systems $=710 \mathrm{pg} / \mathrm{mL}^{7}$ vs BioVendor $=2,330 \mathrm{pg} / \mathrm{mL}$ in this study), possibly owing to the difference in ELISA used.
In this study and our previous study on FGF-21, ${ }^{2}$ we found no correlation with the total or subcategory NMDAS scores for patients who completed this rating scale at the time of recruitment. This is in contrast to other studies that have found moderate to strong correlations between biomarker concentrations and disease severity measured using the NMDAS and the Japanese Mitochondrial Disease Rating Scale. ${ }^{6,72,15}$ In the European study, a correlation was identified between GDF-15 and NMDAS-rated severity for asymptomatic patients, moderately affected patients, or moderately affected patients $\left(r_{s}=0.54, p<0.001\right)$, but not for severely affected patients. ${ }^{6}$ This disparity may be due to the method of analysis, as it was reported that a parametric correlation test (Pearson) was used for the severe group and then compared to a nonparametric correlation test (Spearman) for asymptomatic, mild and moderate severity groups. A lack of correlation between serum biomarker concentration and disease progression may indicate that the current means of assessing disease progression are inadequate or too narrow for the phenotypic diversity of these disease groups. Alternatively, the lack of association may indicate the inability of these biomarkers to infer disease progression on the basis of being surrogate markers and not mediators of disease, a detail that warrants further investigation. Finally, the single follow-up sampling of both FGF-21 and GDF-15 in the European m.3243A $>$ G cohort, 2 years after the initial sample, may not provide adequate frequency or time scales for these markers to reliably track disease progression in combination with the NMDAS. ${ }^{6,12}$ Estimation of progression may therefore be improved by more frequent sampling in addition to more sensitive means of assessing severity. Thus, larger studies with more sensitive clinical tools to measure disease progression, performed over longer follow-up periods and with more frequent sampling points, may be required to clarify whether biomarkers such as GDF-15 and FGF-21 are able to indicate disease progression.

The clinical diagnostic outlook for mitochondrial disease has been dramatically improved over the past 5 years with the identification of FGF-21 as a useful indicator of muscle manifesting mitochondrial disease and now more so with GDF-15 as a more sensitive and broadly indicative marker of mitochondrial disease. Continued investigation into the pathophysiologic relationship of these markers with mitochondrial disease may strengthen their diagnostic utility or identify better candidate biomarkers. In any case, front-line diagnostic indicator tests, such as serum GDF-15 concentration, coupled with exhaustive genetic sequencing methods, afforded by advances in next-generation sequencing technologies, herald a potentially new 
age for mitochondrial disease diagnosis. ${ }^{13}$ It is hoped that the clinical, fiscal, and personal burden of mitochondrial diseases may be improved through better management and treatment resulting from improved diagnostic capabilities.

\section{AUTHOR CONTRIBUTIONS}

Statistical analysis was carried out by R.L.D. with assistance from J. Patterson (both from the Kolling Institute of Medical Research, St. Leonards, Australia). Dr. R.L.D. conceptualized and designed the study, acquired data, analyzed data, carried out statistical analyses, interpreted data, drafted the manuscript, and revised the manuscript. Dr. C.L. acquired data and revised the manuscript. Professor C.M.S. designed and funded the study, interpreted data, and revised the manuscript.

\section{ACKNOWLEDGMENT}

R.L.D. is a NHMRC early career postdoctoral fellow, C.L. is a NHMRC postgraduate scholar, and C.M.S. is a NHMRC practitioner fellow. The authors thank Dr. J. Patterson and Dr. A. Dona for their discussion of statistical analyses, as well as the patients and their referring doctors for participation.

\section{STUDY FUNDING}

No targeted funding reported.

\section{DISCLOSURE}

The authors report no disclosures relevant to the manuscript. Go to Neurology.org for full disclosures.

Received December 27, 2015. Accepted in final form February 21, 2016

\section{REFERENCES}

1. Clarke C, Xiao R, Place E, et al. Mitochondrial respiratory chain disease discrimination by retrospective cohort analysis of blood metabolites. Mol Genet Metab 2013;110: 145-152.

2. Davis RL, Liang C, Edema-Hildebrand F, et al. Fibroblast growth factor 21 is a sensitive biomarker of mitochondrial disease. Neurology 2013;81:1819-1826.

3. Suomalainen A, Elo JM, Pietilainen KH, et al. FGF-21 as a biomarker for muscle-manifesting mitochondrial respira- tory chain deficiencies: a diagnostic study. Lancet Neurol 2011;10:806-818.

4. Tyynismaa H, Carroll CJ, Raimundo N, et al. Mitochondrial myopathy induces a starvation-like response. Hum Mol Genet 2010;19:3948-3958.

5. Kalko SG, Paco S, Jou C, et al. Transcriptomic profiling of TK2 deficient human skeletal muscle suggests a role for the p53 signalling pathway and identifies growth and differentiation factor-15 as a potential novel biomarker for mitochondrial myopathies. BMC Genomics 2014;15:91.

6. Koene $\mathrm{S}$, de Laat $\mathrm{P}$, van Tienoven $\mathrm{DH}$, et al. Serum GDF15 levels correlate to mitochondrial disease severity and myocardial Strain, but not to disease progression in adult m.3243A $>$ G carriers. JIMD Rep 2015;24:69-81.

7. Yatsuga S, Fujita Y, Ishii A, et al. Growth differentiation factor 15 as a useful biomarker for mitochondrial disorders. Ann Neurol 2015;78:814-823.

8. Fujita Y, Ito M, Kojima T, et al. GDF15 is a novel biomarker to evaluate efficacy of pyruvate therapy for mitochondrial diseases. Mitochondrion 2015;20:34-42.

9. Walker UA, Collins S, Byrne E. Respiratory chain encephalomyopathies: a diagnostic classification. Eur Neurol 1996;36:260-267.

10. Schaefer AM, Phoenix C, Elson JL, et al. Mitochondrial disease in adults: a scale to monitor progression and treatment. Neurology 2006;66:1932-1934.

11. Medical Research Council of the United Kingdom. Aids to Examination of the Peripheral Nervous System. Palo Alto, CA: Pendragon House; 1978.

12. Koene $S$, de Laat $P$, van Tienoven $D H$, et al. Serum FGF21 levels in adult m.3243A $>$ G carriers: clinical implications. Neurology 2014;83:125-133.

13. Liang C, Ahmad K, Sue CM. The broadening spectrum of mitochondrial disease: shifts in the diagnostic paradigm. Biochim Biophys Acta 2014;1840:1360-1367.

14. Ho JE, Mahajan A, Chen $\mathrm{MH}$, et al. Clinical and genetic correlates of growth differentiation factor 15 in the community. Clin Chem 2012;58:1582-1591.

15. Yatsuga S, Povalko N, Nishioka J, et al. MELAS: a nationwide prospective cohort study of 96 patients in Japan. Biochim Biophys Acta 2012;1820:619-624.

\section{WriteClick ${ }^{\circledR}$ rapid online correspondence}

Have a comment on a recent Neurolog $y^{\circledR}$ article you would like to share? Now it is easier and more convenient. Neurology.org has launched WriteClick on the home page and sidebars of each article to encourage remarks and debate among users.

WriteClick is restricted to comments about studies published in Neurology within the last eight weeks.

Learn more at Neurology.org/letters 


\section{Neurology}

\section{A comparison of current serum biomarkers as diagnostic indicators of mitochondrial diseases}

Ryan L. Davis, Christina Liang and Carolyn M. Sue

Neurology 2016;86;2010-2015 Published Online before print April 27, 2016

DOI 10.1212/WNL.0000000000002705

\section{This information is current as of April 27, 2016}

\section{Updated Information \& Services}

References

Citations

Subspecialty Collections

Permissions \& Licensing

Reprints including high resolution figures, can be found at: http://n.neurology.org/content/86/21/2010.full

This article cites 14 articles, 4 of which you can access for free at: http://n.neurology.org/content/86/21/2010.full\#ref-list-1

This article has been cited by 4 HighWire-hosted articles: http://n.neurology.org/content/86/21/2010.full\#\#otherarticles

This article, along with others on similar topics, appears in the following collection(s):

\section{Class III}

http://n.neurology.org/cgi/collection/class_iii

Cohort studies

http://n.neurology.org/cgi/collection/cohort_studies

Diagnostic test assessment

http://n.neurology.org/cgi/collection/diagnostic_test_assessment Mitochondrial disorders; see Genetics/Mitochondrial disorders http://n.neurology.org/cgi/collection/mitochondrial_disorders_see_gene tics-mitochondrial_disorders

Information about reproducing this article in parts (figures,tables) or in its entirety can be found online at:

http://www.neurology.org/about/about_the_journal\#permissions

Information about ordering reprints can be found online:

http://n.neurology.org/subscribers/advertise

Neurology ${ }^{\circledR}$ is the official journal of the American Academy of Neurology. Published continuously since 1951 , it is now a weekly with 48 issues per year. Copyright @ 2016 American Academy of Neurology. All rights reserved. Print ISSN: 0028-3878. Online ISSN: 1526-632X.

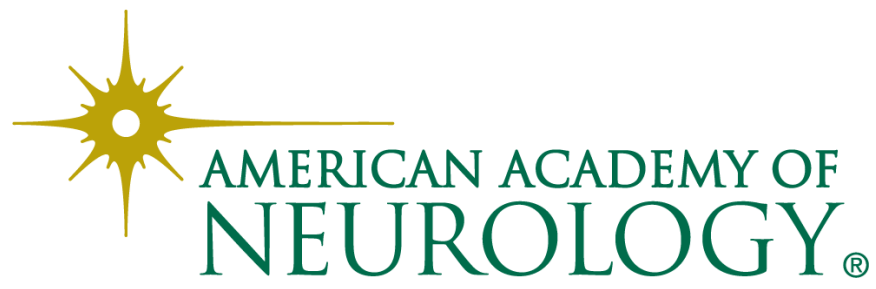

